\title{
Contribution of fuzziness and uncertainty to modern artificial intelligence
}

On the 31st August 1955, J. McCarthy (Dartmouth College, New Hampshire), M.L. Minsky (Harvard University), N. Rochester (I.B.M. Corporation) and C.E. Shannon (Bell Telephone Laboratories) proposed a meeting to a group of researchers to be held in the summer of 1956 in order to provide ideas on each aspect of learning and each feature of intelligence capable of being simulated on machines. During the encounter, later known as the Dartmouth Conference, the term artificial intelligence (AI) was coined.

In 2006, the 50th anniversary of the Dartmouth Conference has been celebrated worldwide. Despite the time elapsed during these 50 years, many problems about finding the precise descriptions of the features of the brain and the mind mentioned in the 1955 proposal still remain open today. With this idea in mind, the international conference 50 Years of Artificial Intelligence: Campus in Multidisciplinary Perception and Intelligence was held in Albacete (Spain), July 10-14, 2006. The idea was to gather researchers from different areas in AI, Computer Science, Perception, Logic, Philosophy, Linguistics, Neurobiology, etc., with the aim of recovering the enthusiastic atmosphere which was present in the first year of AI and foster scientific exchanges among all these areas.

This special section aims at presenting a selection of the papers proposed under the title Contribution of Fuzziness and Uncertainty to Modern Artificial Intelligence relating the different areas of AI with Fuzzy Set theory and Fuzzy logic. On behalf of the authors, the editor of this special section would like to express his gratitude to the reviewers and the Fuzzy Sets and Systems Co-Editors-in-Chief, B. De Baets and D. Dubois for their support.

Antonio Fernández-Caballero Departamento de Sistemas Informáticos, Instituto de Investigación en Informática de Albacete (I3A) Universidad de Castilla-La Mancha, 02071 Albaute, Spain E-mail address: caballer@dsi.uclm.es 